\title{
Large deviation tail estimates and related limit laws for stochastic fixed point equations
}

Collamore, Jeffrey F.; Vidyashankar, Anand N.

Published in:

Random Matrices and Iterated Random Functions

Publication date:

2013

Document version

Peer reviewed version

Citation for published version (APA):

Collamore, J. F., \& Vidyashankar, A. N. (2013). Large deviation tail estimates and related limit laws for stochastic fixed point equations. In M. Lowe, \& G. Alsmeyer (Eds.), Random Matrices and Iterated Random Functions (Vol. 63, pp. 91-117). Springer. 
From Random Matrices and Iterated Random Functions (Alsmeyer, Löwe, eds.), Springer, pp. 91-117.

\title{
Large Deviation Tail Estimates and Related Limit Laws for Stochastic Fixed Point Equations
}

\author{
Jeffrey F. Collamore and Anand N. Vidyashankar
}

\begin{abstract}
We study the forward and backward recursions generated by a stochastic fixed point equation (SFPE) of the form $V \stackrel{d}{=} A \max \{V, D\}+B$, where $(A, B, D) \in$ $(0, \infty) \times \mathbb{R}^{2}$, for both the stationary and explosive cases. In the stationary case (when $\mathbf{E}[\log A]<0$ ), we present results concerning the precise tail asymptotics for the random variable $V$ satisfying this SFPE. In the explosive case (when $\mathbf{E}[\log A]>0$ ), we establish a central limit theorem for the forward recursion generated by the SFPE, namely the process $V_{n}=A_{n} \max \left\{V_{n-1}, D_{n}\right\}+B_{n}$, where $\left\{\left(A_{n}, B_{n}, D_{n}\right): n \in \mathbb{Z}_{+}\right\}$is an i.i.d. sequence of random variables. Next, we consider recursions where the driving sequence of vectors, $\left\{\left(A_{n}, B_{n}, D_{n}\right): n \in \mathbb{Z}_{+}\right\}$, is modulated by a Markov chain in general state space. We demonstrate an asymmetry between the forward and backward recursions and develop techniques for estimating the exceedance probability. In the process, we establish an interesting connection between the regularity properties of $\left\{V_{n}\right\}$ and the recurrence properties of an associated $\xi$-shifted Markov chain. We illustrate these ideas with several examples.
\end{abstract}

\section{Introduction}

In this article, we consider stochastic fixed point equations (SFPE) of the form

Jeffrey F. Collamore

Department of Mathematical Sciences, University of Copenhagen, Universitetsparken 5, DK-2100 Copenhagen $\varnothing$, Denmark. e-mail: collamore@math.ku.dk

Research supported in part by Danish Research Council (SNF) Grant No. 09-092331.

Anand N. Vidyashankar

Department of Statistics, Volgeneau School of Engineering, George Mason University,

Fairfax, VA 22030, U.S.A. e-mail: avidyash@gmu.edu

Research supported in part by NSF grant DMS 1107108. 


$$
V \stackrel{d}{=} f(V)
$$

where $f$ is a known random function and $V$ is an unknown random variable in $\mathbb{R}$, independent of $f$. Such equations arise in a variety of applications, ranging from collective risk theory, queuing theory, financial time series modeling, and life insurance mathematics, to problems in branching processes and computer science. In these applications, it is often of interest to describe the tail behavior of the random variable $V$ in (1).

Early work on this problem can be traced to the celebrated paper of Kesten [22], who considered the linear recursion

$$
V \stackrel{d}{=} A V+B, \quad(A, B) \in \mathbb{R}^{2},
$$

in a higher dimensional setting, and applied the results to describe the tail behavior of certain martingale limits that arise in multi-type branching processes in random environments. In this context, he showed that if $\mathbf{E}[\log A]<0$ (hereafter referred to as the stationary case) and appropriate regularity conditions are satisfied, then

$$
\mathbf{P}\{V>u\} \sim C u^{-\xi} \quad \text { as } \quad u \rightarrow \infty,
$$

where $\xi$ is the nonzero solution to the equation $\mathbf{E}\left[A^{\xi}\right]=1$. This result was later extended in $\mathbb{R}^{1}$ to more general recursions by Goldie [18]; see Section 2.1 below.

Identifying and characterizing the constant $C$ in (3) is much more of a delicate affair compared to the problem of characterising $\xi$. While $\xi$ only depends on the multiplicative factor $A$ of the given recursion, the value of the constant $C$ depends on the pair $(A, B)$ in (2) or, more generally, on the function $f$ in (1). For the linear recursion (2), a nonrigorous approach — following earlier work by Yakin and Pollak [34] on likelihood ratio testing and sequential change point problems in statisticswas suggested in [32]. Quite recently, a rigorous solution was provided in [16] for the linear recursion (2) and independent random variables $A$ and $B$ using a coupling argument. A rigorous probabilistic solution - which holds for a general class that subsumes the models considered in Goldie [18] — was recently developed by the authors in [11].

In this article, we begin by giving a characterization of the constant $C$ in the stationary case for the SFPE $V \stackrel{d}{=} A \max \{V, D\}+B$ and its extension to random maps. Next, we study the forward recursion $V_{n}:=A_{n} \max \left\{V_{n-1}, D_{n}\right\}+B_{n}$ in the explosive case; that is, when $\mathbf{E}[\log A]>0$. We show that $\left(V_{n} / P_{n}\right) \rightarrow \mathscr{W}$ as $n \rightarrow \infty$ w.p. 1 for a certain random variable $P_{n}$ and establish a central limit theorem for $V_{n}$. Finally, we provide a nontrivial extension of the results in [11] to the Markov case; that is, the case when $\left\{\left(A_{n}, B_{n}, D_{n}\right): n=1,2, \ldots\right\}$ is a Markov sequence and $V_{n}:=A_{n} \max \left\{V_{n-1}, D_{n}\right\}+B_{n}$ (or a related forward or backward recursion as described in Sections 2.1 and 3.1 below). While Markovian extensions of Goldie's [18] result have been considered in [30] for the linear recursion (2) and in [8] for a wider class of backward recursions, a unified treatment encompassing an estimate for the pair $(C, \xi)$ has not been systematically given. Here we present a unified ap- 
proach, which builds upon work developed by the authors in [11] and earlier work of one of the authors in [8]. A key idea that facilitates this unification is the observation that, if the sequence $\left\{\left(A_{n}, B_{n}\right): n=1,2, \ldots\right\}$ possesses a regenerative structure, then the process $\left\{V_{n}: n=0,1, \ldots\right\}$ inherits this regenerative property and the original forward recursion of the SFPE can be expressed as a forward recursion of another SFPE (but belonging to the same class of SFPEs under investigation); i.e., a forward recursion with a different driving sequence. A similar result also holds for the case of backward sequences. Expectedly, the driving sequence will now involve the regeneration times of the modulating Markov sequence.

We illustrate our results with a variety of examples drawn from insurance, financial mathematics, branching processes, and statistical inference.

\section{Recursions driven by i.i.d. sequences}

\subsection{The stationary case}

Our starting point is the SFPE

$$
V \stackrel{d}{=} f(V) \equiv F_{Y}(V)
$$

where $F_{Y}(V) \equiv F(V, Y)$ for some deterministic function $F: \mathbb{R} \times \mathbb{R}^{d} \rightarrow \mathbb{R}$, assumed throughout the article to be measurable and to be continuous in its first component. In this representation, the random function $f$ is determined by an environmental random vector $Y$ and independent of $V$. Moreover, we implicitly assume the shape condition

$$
F_{Y}(v)=A v+o(v) \text { a.s. as } v \rightarrow \infty,
$$

where $A$ takes values on the positive real axis. In the following discussion, we will assume without loss of generality that $Y=\left(\log A, Y^{\prime}\right)$ for some $Y^{\prime} \in \mathbb{R}^{d-1}$.

To assure that (4) has a stationary solution, we need the multiplicative factor $A$ in (5) to be contracting; that is, $\mathbf{E}[\log A]<0$. Let $\lambda(\alpha):=\mathbf{E}\left[A^{\alpha}\right]$ and $\Lambda(\alpha):=\log \lambda(\alpha)$ denote the moment generating function and the cumulant generating function of the random variable (r.v.) $\log A$, respectively, where $\alpha \in \mathbb{R}$. Also, let $\mu_{A}$ denote the probability distribution of $A$. For any function $g$, let $\operatorname{dom}(g)$ denote the domain of $g$. We assume

$$
\mathbf{E}\left[A^{\xi}\right]=1, \quad \text { for some } \xi \in(0, \infty) \cap \operatorname{dom} \Lambda^{\prime} .
$$

To determine the tail behavior of $V$, one approach is to observe that the SFPE (4) induces a renewal equation. Namely,

$$
e^{\xi_{v}} \mathbf{P}\left\{V>e^{v}\right\}=e^{\xi^{v}}\left\{\mathbf{P}\left\{V>e^{v}\right\}-\mathbf{P}\left\{A V>e^{v}\right\}\right\}+e^{\xi v} \int_{\mathbb{R}} \mathbf{P}\left\{V>e^{v-x}\right\} \mu_{A}(d x) .
$$


Hence, setting $Z(v)=e^{\xi v} \mathbf{P}\left\{V>e^{v}\right\}$ and $z(v)$ equal to the first term on the righthand side of the previous equation, we obtain that

$$
Z(v)=z(v)+Z * \mu_{A, \xi}(v), \text { where } \mu_{A, \xi}(d x)=e^{\xi x} \mu_{A}(d x),
$$

which is closely related to the renewal equation. Thus, if we knew that the function $z$ were directly Riemann integrable, then the renewal theorem could be invoked to obtain that $Z(v) \rightarrow C$ as $v \rightarrow \infty$ and, consequently, $\mathbf{P}\{V>u\} \sim C u^{-\xi}$ as $u \rightarrow \infty$.

Typically, it is impossible to verify that $z$ is directly Riemann integrable. However, this assumption can be avoided by using a smoothing argument introduced in [18]. This techniques yields the following very general theorem, proved by Goldie ([18], Theorem 2.3), building upon the previous work of Kesten [22].

Theorem 1 Assume that there exists a nonnegative random variable A which is nonarithmetic and satisfies (6), and assume that

$$
\mathbf{E}\left[\left|\left(f(V)^{+}\right)^{\xi}-\left((A V)^{+}\right)^{\xi}\right|\right]<\infty .
$$

Then

$$
\lim _{u \rightarrow \infty} u^{\xi} \mathbf{P}\{V>u\}=C,
$$

where

$$
C=\frac{1}{\xi \lambda^{\prime}(\xi)} \mathbf{E}\left[\left(f(V)^{+}\right)^{\xi}-\left((A V)^{+}\right)^{\xi}\right] .
$$

While this estimate is easily obtained from the renewal theorem under weak assumptions, this approach has certain limitations. For instance, it is not possible to establish the finiteness and positivity of the constant $C$ without further assumptions. Furthermore, the expression for $C$ in (9) is defined in terms of $V$ and, thus, is not particularly fruitful in practical problems. A useful characterization in terms of the forward process $\left\{V_{n}\right\}$ in (10) below would, in particular, facilitate statistical inference and numerical procedures such as importance sampling.

To address the above difficulties associated with (9), an alternative approach was recently developed in Collamore and Vidyashankar [11]. This approach is based on the observation that the process $V_{n}:=F_{Y_{n}}\left(V_{n-1}\right)$ (obtained via forward iterations of the SFPE, see below) is Markovian and behaves like a multiplicative random walk for large values of $V_{n-1}$. Thus, we may use nonlinear renewal theory to characterize this process for "large" $V_{n-1}$, and then adapt methods from Markov chain theory to quantify the discrepancy between these two processes.

To describe this approach, we first need to introduce the forward and backward sequences generated by a given SFPE. Let $\left\{Y_{n}: n=1,2, \ldots\right\}$ be an i.i.d. sequence having the same probability law as $Y$ in (4). The forward sequence $\left\{V_{n}\right\}$ is defined by

$$
V_{n}(v)=F_{Y_{n}} \circ F_{Y_{n-1}} \circ \cdots \circ F_{Y_{1}}(v), \quad n=1,2, \ldots, \quad V_{0}=v ;
$$

while the backward sequence $\left\{Z_{n}\right\}$ is defined by

$$
Z_{n}(z)=F_{Y_{1}} \circ F_{Y_{2}} \circ \cdots \circ F_{Y_{n}}(z), \quad n=1,2, \ldots, \quad Z_{0}=z .
$$


The Furstenberg-Letac principle states that-although the sample paths of the forward and backward sequences are manifestly different-the forward sequence converges in distribution to a random variable $V$ provided that the backward sequence converges a.s. to a random variable $Z$ and is independent of the initial value; furthermore, the distributions of $V$ and $Z$ are the same ([17], [23]). This leads to the issue of determining which sequence - the forward or backward sequence-is more amenable for analysis, and this, of course, is problem-dependent. In Collamore and Vidyashankar [11], it is suggested that the forward sequence is preferable for understanding the tail behavior of $V$ described by Theorem 2.1, and this approach also appears advantageous for the Monte Carlo simulation of these probabilities ( $c f$. [9]). Generally speaking, the advantage of the forward sequence is that this process is a recurrent Markov chain and hence has useful ergodic properties (while the backward sequence converges a.s., which is useful when analytic, rather than probabilistic, methods are employed).

We now specialize to the quasi-linear recursion

$$
V \stackrel{d}{=} F_{Y}(V), \quad F_{Y}(v)=A \max \{v, D\}+B,
$$

where $Y \equiv(\log A, B, D) \in \mathbb{R}^{3}$. This SFPE is often called "Letac's Model E" and, as we will see in Section 2.3, has a wide applied relevance. This class of models is roughly equivalent to the class considered by Goldie in [18].

First introduce the following regularity conditions.

\section{Hypotheses:}

$\left(H_{0}\right)$ The random variable $A$ has an absolutely continuous component with respect to Lebesgue measure with a nontrivial density in a neighborhood of $\mathbb{R}$.

$\left(H_{1}\right) \Lambda(\xi)=0$ for some $\xi \in(0, \infty) \cap \operatorname{dom}\left(\Lambda^{\prime}\right)$.

$\left(H_{2}\right) \mathbf{E}\left[|B|^{\xi}\right]<\infty$ and $\mathbf{E}\left[(A|D|)^{\xi}\right]<\infty$.

( $\left.H_{3}\right) \mathbf{P}\{A>1, B>0\}>0$ or $\mathbf{P}\{A>1, B \geq 0, D>0\}>0$.

Let $P_{V}$ denote the transition kernel of $\left\{V_{n}\right\}$. Also, let $\mathscr{B}\left(\mathbb{R}^{d}\right)$ denote the Borel sets on $\mathbb{R}^{d}, d \geq 1$. Then under Hypotheses $\left(H_{0}\right),\left(H_{1}\right)$, and $\left(H_{2}\right)$, the forward process $\left\{V_{n}\right\}$ is a Markov chain satisfying the minorization condition

$$
\delta \mathbf{1}_{\mathscr{C}}(v) v(E) \leq P_{V}(v, E), \quad v \in \mathbb{R}, E \in \mathscr{B}(\mathbb{R}),
$$

where $\delta$ is a positive constant, $\mathscr{C}$ a nontrivial set in $\mathbb{R}$, and $v$ a probability measure; see [11], Lemma 5.1. Hence by a classical result of Athreya and Ney [4] and Nummelin [28], it follows that the forward process $\left\{V_{n}\right\}$ admits a regeneration structure. More precisely, we can find a sequence of independent times $0 \leq T_{0}<T_{1}<\cdots$ such that:

(i) $\tau_{i}:=T_{i}-T_{i-1}$ is an i.i.d. sequence, $i \in \mathbb{Z}_{+}$;

(ii) $\left\{V_{T_{i-1}}, \ldots, V_{T_{i}-1}\right\}$ form independent blocks, $i \in \mathbb{Z}_{+}$;

(iii) $V_{T_{i}} \sim v$, independent of its past. 
Let $\tau$ denote a typical regeneration time, that is to say, the first regeneration time assuming that regeneration has occurred at time zero. Then by [29], p. 75, it follows as a consequence of the regeneration lemma that

$$
\mathbf{P}\{V>u\}=\frac{\mathbf{E}\left[N_{u}\right]}{\mathbf{E}[\tau]}, \quad \text { where } \quad N_{u}:=\sum_{n=0}^{\tau-1} \mathbf{1}_{(u, \infty)}\left(V_{n}\right) .
$$

In particular, $N_{u}$ counts the number of exceedances of $\left\{V_{n}\right\}$ occurring over a single regeneration cycle, and this number tends to zero as $u \rightarrow \infty$. Thus $\left\{N_{u}>u\right\}$ is a rare event, and quantifying $\mathbf{E}\left[N_{u}\right]$ for large $u$ is a large deviation problem. It is natural to characterize this probability using a change of measure of the driving sequence $\left\{Y_{n}\right\}$ in (12).

Let $\mu$ denote the probability law of $Y \equiv(\log A, B, D)$, and let $\xi$ be given as in (6), and define

$$
\mu_{\xi}(E)=\int_{E} e^{\xi x} d \mu(x, y, z), \quad E \in \mathscr{B}\left(\mathbb{R}^{3}\right) .
$$

Then $\mu_{\xi}$ is itself a probability measure and, with respect to this measure, we easily obtain that the process $\left\{V_{n}\right\}$ is transient ([11], Lemma 5.2). Set $\mathscr{T}_{u}=\inf \left\{n: Y_{n}>u\right\}$, and consider the dual change of measure:

$$
\mathfrak{L}\left(\log A_{n}, B_{n}, D_{n}\right)= \begin{cases}\mu_{\xi} & \text { for } n=1, \ldots, \mathscr{T}_{u}, \\ \mu & \text { for } n>\mathscr{T}_{u} .\end{cases}
$$

Let $\mathbf{E}_{\mathfrak{D}}[\cdot]$ denote the expectation with respect to this dual measure and $\mathbf{E}_{\xi}[\cdot]$ denote the expectation with respect to $\mu_{\xi}$.

To estimate $\mathbf{E}_{\mathfrak{D}}\left[N_{u}\right]$, it is helpful to observe that this expectation splits into two parts, one describing the "short term" behavior over a regeneration cycle, and the other describing the "long term" behavior. By [11], Proposition 6.1, conditional on $V_{0} \sim v$, we have that as $u \rightarrow \infty$,

$$
\mathbf{E}\left[N_{u}\right] \sim \mathbf{E}_{\xi}\left[W^{\xi} \mathbf{1}_{\{\tau=\infty\}}\right] \cdot u^{-\xi} \mathbf{E}_{\mathfrak{D}}\left[N_{u}\left(\frac{V_{T_{u}}}{u}\right)^{-\xi}\right],
$$

where $W:=\lim _{n \rightarrow \infty} V_{n} /\left(A_{1} \cdots A_{n}\right)$. It is worth noting that in a wide variety of examples, including all of the examples in Section 2.3 below, the random variable $W$ reduces to the perpetuity sequence

$$
W=V_{0}+\frac{B_{1}}{A_{1}}+\frac{B_{2}}{A_{1} A_{2}}+\cdots,
$$

which, in (16), is killed upon regeneration of the transient process $\left\{V_{n}\right\}$.

Notice that under the shape condition (5), the process $\left\{V_{n}\right\}$ resembles a multiplicative random walk when this process is far away from the origin. Moreover, the exceedance probabilities for the multiplicative random walk are well-known from classical risk theory ( $c f .[2])$ and for perturbed random walks from nonlinear renewal theory (cf. [33]). Trivially, in (16), 


$$
\left(V_{T_{u}} / u\right)^{-\xi}=\exp \left\{-\xi\left(\log V_{T_{u}}-\log u\right)\right\},
$$

where the exponent on the right-hand side describes the overjump of the perturbed random walk $\left\{\log \left(V_{n} \vee 1\right)\right\}$ over a barrier at level $\log u$. Consequently, using extensions of results from [33], the second quantity on the right-hand side of (16) can be identified, as $u \rightarrow \infty$, as $u^{-\xi} \mathbf{E}\left[N_{u}^{*}\right]$, where $N_{u}^{*}$ denotes the number of exceedances above level $\log u$ which occur for the random walk $S_{n}=\sum_{i=1}^{n} \log A_{i}$ over its regeneration cycle, that is to say, over a cycle starting at the origin and continuing until time $\tau^{*}=\inf \left\{n: S_{n} \leq 0\right\}$. Thus, the first term on the right of (16) describes the discrepancy between the decay constant arising for the process $\left\{V_{n}\right\}$ and that arising for the corresponding multiplicative random walk.

To make these ideas rigorous, set $A_{0}=1$ and $B_{0} \sim v$ (where $v$ is given as in $(\mathscr{M}))$. Now define the perpetuity sequence associated with $\left\{V_{n}\right\}$ by

$$
Z_{n}^{(p)}=\sum_{i=0}^{n} \frac{B_{i}}{A_{0} \cdots A_{i}}, \quad n=0,1, \ldots,
$$

and define the conjugate sequence associated with $\left\{V_{n}\right\}$ by

$$
Z_{n}^{(c)}=\min \left\{Z_{n}^{(p)}, 0, \bigwedge_{k=1}^{n} \sum_{i=0}^{k-1} \frac{B_{i}}{A_{0} \cdots A_{i}}-\frac{D_{k}}{A_{0} \cdots A_{k-1}}\right\} .
$$

It is easily seen that both of these quantities are backward sequences in the sense of (11); specifically, (17), resp. (18) are generated by the recursions

$$
F_{Y}^{(p)}(v)=\frac{v}{A}+\frac{B}{A} \quad \text { and } \quad F_{Y}^{(c)}=\frac{1}{A} \min \{\check{D}, v\}+\frac{B}{A},
$$

where $\breve{D}_{0}:=-B_{0}$ and $\breve{D}_{i}:=-A_{i} D_{i}-B_{i}$ for $i=1,2, \ldots$

Our main result in this section is the following:

Theorem 2 Assume (12), and suppose that $\left(H_{0}\right),\left(H_{1}\right),\left(H_{2}\right)$, and $\left(H_{3}\right)$ are satisfied. Then

$$
\lim _{u \rightarrow \infty} u^{\xi} \mathbf{P}\{V>u\}=C
$$

for a finite positive constant $C$. Moreover, $C=\lim _{n \rightarrow \infty} C_{n}$, where

$$
C_{n}=\frac{1}{\xi \lambda^{\prime}(\xi) \mathbf{E}[\tau]} \mathbf{E}_{\xi}\left[\left(\left(Z_{n}^{(p)}-Z_{n}^{(c)}\right)^{+}\right)^{\xi} \mathbf{1}_{\{\tau>n\}}\right],
$$

and $\mathfrak{R}_{n}:=C-C_{n}=o\left(e^{-\varepsilon n}\right)$ as $n \rightarrow \infty$, for some $\varepsilon>0$.

For the proof of Theorem 2, see [11]. In particular, nonnegativity of the constant follows immediately from $\left(\mathrm{H}_{3}\right)$ and the fact that zero is contained in the collection minimized on the right-hand side of (18).

As demonstrated in [11], this method generalizes to a number of related problems. For example, it is shown that the method yields a useful upper bound, akin 
to the Lundberg inequality from insurance mathematics. Moreover, the method provides a simple characterization for the extremal index of $\left\{V_{n}\right\}$ (thus producing a considerable simplification of that developed for the special case of the ARCH(1) process in [19]). For details, see [11], Section 2.3.

Finally, the results can be generalized to a wider class of SFPEs. The main assumptions needed are the presence of a cancellation condition, namely

$$
A \max \left\{v, D^{*}\right\}+B^{*} \leq f(v) \leq A \max \{v, D\}+B,
$$

together with the Lipschitz condition

$$
\sup _{v \neq w} \frac{|f(v)-f(w)|}{|v-w|}=L, \quad \mathbf{E}[\log L]<0,
$$

where the approximating Letac models appearing in the cancellation condition are assumed to satisfy $\left(H_{0}\right)-\left(H_{3}\right)$. Then we obtain a complete analog of Theorem 2.2, although the constant $C$ is now expressed recursively and hence does not assume a simple analytical form. For details, see [11], Section 2.4.

\subsection{The explosive case}

While the previous section was concerned with the stationary case, namely when $\mathbf{E}[\log A]<0$, in this section we present new results for the case $\mathbf{E}[\log A] \geq 0$. Note by Jensen's inequality that $\mathbf{E}[A] \geq 1$, and by the non-degeneracy of $A$ it follows that $\mathbf{E}[A]>1$. Our first result concerns the distributional behavior of $\log V_{n}$ when $1<\mathbf{E}[A]<\infty$. In the following, we denote the variance of a random variable $A$ by $\operatorname{Var}(A)$.

Theorem 3 Let $\left\{V_{n}\right\}$ denote the forward process generated by Letac's Model E, as given in (10) and (12). Further assume that $\mathbf{E}[\log (|B| / A)]<\infty, \mathbf{E}[\log |D|]<\infty$, and $\mathbf{E}[\log A]>0$. Then:

(i) $V_{n}$ diverges to infinity w.p. 1 .

(ii) Setting $\mu=\mathbf{E}[\log A], \sigma^{2}=\operatorname{Var}(\log A)$, and $\mathscr{R}_{n}=n^{-\frac{1}{2}} \sigma^{-1}\left\{\log \left(V_{n}\right)-n \mu\right\}$, then $\left\{\mathscr{R}_{n}\right\}$ converges in distribution to a standard normal distribution as $n \rightarrow \infty$.

Proof. Using the forward recursion, we can express $V_{n}$ as follows:

$$
V_{n}=\left(A_{1} \cdots A_{n}\right) \max \left\{J_{1, n}, J_{2, n}\right\},
$$

where

$$
J_{1, n}=\sum_{i=0}^{n} \frac{B_{i}}{A_{1} \cdots A_{i}}
$$

and 


$$
J_{2, n}=\bigvee_{k=1}^{n}\left[\sum_{i=k}^{n} \frac{B_{i}}{A_{1} \cdots A_{i}}+\frac{D_{k}}{A_{1} \cdots A_{k-1}}\right] \equiv \bigvee_{k=1}^{n} J_{2, n, k}
$$

Now, by taking logarithms on both sides of (21), we get that

$$
\mathscr{R}_{n}=\frac{\sum_{j=1}^{n}\left(\log A_{j}-\mu\right)}{\sqrt{n} \sigma}+\frac{\log \max \left\{J_{1, n}, J_{2, n}\right\}}{\sqrt{n} \sigma} .
$$

It is worthwhile to notice that the second term is well-defined since, by our assumptions, $V_{n}>0$ and $\prod_{i=1}^{n} A_{i}>0$. To complete the proof, we need to show that the second term converges to zero in probability.

To this end, we begin by noticing that

$$
J_{1, n}=\sum_{i=0}^{n} \frac{B_{i}}{A_{i}} \cdot \frac{1}{A_{1} \cdots A_{i-1}} .
$$

Under our assumptions, $J_{1, n}$ is a perpetuity generated by the driving sequence $(B / A, 1 / A)$. Also $\mathbf{E}[\log (1 / A)]<0$. Hence $J_{1, n}$ converges to $J_{1, \infty}$ with probability one, by Theorem 1.3 of [1]. Furthermore, the random variable $J_{1, \infty}$ does not have an atom at zero. Next consider the term $J_{2, n}$. To this end, notice that

$$
\begin{aligned}
J_{2, n, k} & \leq J_{2, n, k}^{+} \equiv \sum_{i=k}^{n} \frac{\left|B_{i}\right|}{A_{1} \cdots A_{i}}+\frac{\left|D_{k}\right|}{A_{1} \cdots A_{k-1}} \\
& \leq \sum_{i=1}^{\infty} \frac{\left|B_{i}\right|}{A_{1} \cdots A_{i}}+\sum_{k=1}^{\infty} \frac{\left|D_{k}\right|}{A_{1} \cdots A_{k-1}} \equiv J
\end{aligned}
$$

where $J<\infty$ w.p. 1 by another application of Theorem 1.3 in [1]. Then

$$
\sup _{n \in \mathbb{Z}_{+}} J_{2, n} \leq J
$$

One can strengthen this bound to a convergence result for $J_{2, n}$ by utilizing

$$
\max _{1 \leq k \leq n}\left\{J_{2, n, k}-J_{2, \infty, k}\right\} \rightarrow 0 \quad \text { w.p. } 1 \text {. }
$$

That is, using standard arguments, one can show that $J_{2, n}$ converges to $J_{2, \infty}$ W.p. 1 , where $J_{2, \infty}=\max _{k} J_{2, k}$ and $J_{2, \infty}$ does not have an atom at 0 . Thus it follows that

$$
\lim _{n \rightarrow \infty} \frac{\log \max \left\{J_{1, n}, J_{2, n}\right\}}{\sqrt{n}}=0
$$

in probability, which completes the proof of the theorem.

From the proof of the above theorem, we can extract the path properties of $\left\{V_{n}\right\}$. We state this as a theorem.

Theorem 4 Let $\left\{V_{n}\right\}$ denote the forward process generated by Letac's Model E, as given in (10) and (12). Further assume that $\mathbf{E}[\log (|B| / A)]<\infty, \mathbf{E}[\log |D|]<\infty$, and 
$\mathbf{E}[\log A]>0$. Then

$$
\lim _{n \rightarrow \infty} \frac{V_{n}}{A_{1} \cdots A_{n}}=V_{\infty} \quad \text { w.p. } 1,
$$

where the limit $V_{\infty}$ is nondegenerate.

The above theorem studies the asymptotic behavior of $\left\{V_{n}\right\}$ under a random normalization instead of a deterministic normalization. Under further strong assumptions, [20] studies the path properties of $\left\{V_{n}\right\}$ under a deterministic normalization. (Consult [20] and references therein for further central limit theorems related to the explosive case.) Extensions of this idea for Letac's Model E are currently being investigated by the authors.

\subsection{Examples and applications}

We now turn to a few examples.

Example 1 The simplest example is the reflected random walk,

$$
W_{n}=\left(X_{n}+W_{n-1}\right)^{+}, \quad n=1,2, \ldots, \quad W_{0}=0,
$$

where $\left\{X_{i}\right\}$ is an i.i.d. sequence of random variables, which is equivalent to the multiplicative process $V_{n}=A_{n} \max \left\{V_{n-1}, 1\right\}$, where $A_{n}=\exp X_{n}$ and $V_{n}=\exp W_{n}$.

Extremes of these processes play a prominant role in queuing theory (cf. [21]) and in collective risk theory. In the classical ruin problem of Lundberg [24] and Cramér [12], one lets $u$ denote the initial capital of the company, $c$ the constant rate of premiums income, and $\left\{\zeta_{i}\right\}$ the i.i.d. claims losses, which are assumed to arise according to a Poisson process, $\left\{N_{t}\right\}$. Then the total capital of the company at time $t$ is given by

$$
Y_{t}=u+c t-\sum_{i=1}^{N_{t}} \zeta_{i}
$$

Now consider the probability of ruin, namely $\Psi(u):=\mathbf{P}\left\{Y_{t}<0\right.$, for some $\left.t \geq 0\right\}$. Using Sparre-Andersen's random walk representation of the risk process together with classical duality, this probability may be equated to $\mathbf{P}\{W>u\}$, where $W:=$ $\lim _{n \rightarrow \infty} W_{n}$ and $X_{i}:=\zeta_{i}-c \tau_{i}$ in (28). For details, see [2].

Example 2 Consider a modification of the previous example, where the insurance company invests its excess capital, earning i.i.d. returns $\left\{R_{n}\right\}$ on these investments. The total capital of the company is then the solution to the recursive sequence of equations

$$
\tilde{Y}_{n}=R_{n} \tilde{Y}_{n-1}-L_{n}, \quad n=1,2, \ldots, \quad \tilde{Y}_{0}=u,
$$

where $L_{n}:=-\left(Y_{n}-Y_{n-1}\right)$ are the discrete-time losses of the insurance business, governed by the Cramér-Lundberg process described in (29). Next, define the dis- 
counted loss process at time $n$ to be the perpetuity sequence

$$
\mathscr{L}_{n}:=\frac{L_{1}}{R_{1}}+\frac{L_{2}}{R_{1} R_{2}}+\cdots+\frac{L_{n}}{R_{1} \cdots R_{n}} .
$$

Then by a simple argument, the probability of ruin is equivalent to $\tilde{\Psi}(u):=$ $\mathbf{P}\left\{\mathscr{L}_{n}>u\right.$, for some $\left.n\right\}$. The process $\left\{\mathscr{L}_{n}\right\}$ is illustrated in Figure 1 and is a backward recursion generated by the function $F_{Y}(v)=v / R+L / R$. In contrast with the

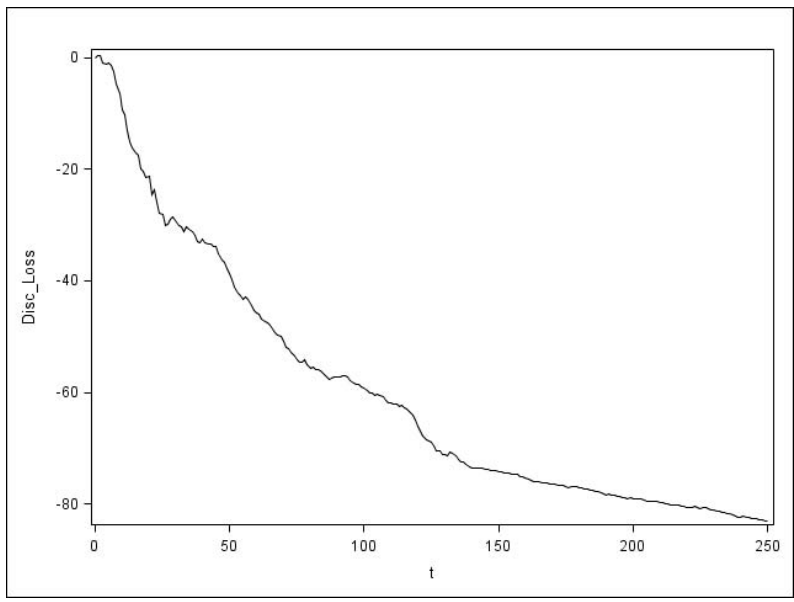

Fig. 1 A sample path of the cumulative loss process. Ruin occurs when this negative-drift process reaches a positive barrier at $u$, where $u$ is the initial capital. This contrasts with the backward process in Example 2.1, which is a multiplicative random walk process.

previous example - whose backward recursion can be shown to be a random walkthe backward process appearing here has dependent increments and is evidently not Markovian.

To determine the probability of ruin, we need to solve for the tail of the r.v. $\mathscr{L} \equiv \sup _{n \in \mathbb{Z}_{+}} \mathscr{L}_{n}$. To this end, observe that $R_{2}^{-1} L_{2}+\cdots+\left(R_{2} \cdots R_{n+1}\right)^{-1} L_{n+1} \stackrel{d}{=} \mathscr{L}_{n}$, and hence by (31),

$$
\mathscr{L}_{n} \stackrel{d}{=} B+A \mathscr{L}_{n-1}, \quad \text { where } \quad A=\frac{1}{R_{1}} \text { and } B=\frac{L_{1}}{R_{1}} .
$$

Now setting $\tilde{\mathscr{L}}=\left(\sup _{n \in \mathbb{Z}_{+}} \mathscr{L}_{n}\right) \vee 0$ yields the SFPE

$$
\tilde{\mathscr{L}} \stackrel{d}{=}(A \tilde{\mathscr{L}}+B)^{+} .
$$

(Alternatively, by a slight variation of this argument, one can also show that $\mathscr{L}$ satisfies the SFPE $\mathscr{L} \stackrel{d}{=} A \max \{\mathscr{L}, 0\}+B$. However, the tail behavior of $\mathscr{L}$ is identical to that of $\tilde{\mathscr{L}}$.) 
As with the previous example, there exists an interesting duality in the sense of Siegmund [31] or Asmussen and Sigman [3]. Namely the process $\left\{\tilde{Y}_{n}\right\}$ is dual to the forward process generated by the SFPE (33); cf. [3], p. 12. While the forward process is Markovian, the process $\left\{\tilde{Y}_{n}\right\}$ is actually studied via the complicated backward process $\left\{\mathscr{L}_{n}\right\}$. As the forward process is simpler than this backward process, it is convenient to first convert the backward process, via its SFPE, into a forward process.

Example 3 Consider a single-type branching process in a random environment. Then the population size at time $n$ is given by

$$
Z_{n}=\left(\sum_{i=1}^{Z_{n-1}} \eta_{n, i}\right)+Q_{n}
$$

where $\left\{\eta_{n, i}: i=1, \ldots, Z_{n-1}\right\}$ represents the number of children in the $n^{\text {th }}$ generation, and $Q_{n}$ represents the number of immigrants in the $n^{\text {th }}$ generation. Assume that the probability laws of these quantities are random, modulated by an i.i.d. environmental sequence $\left\{\zeta_{n}\right\}$. Thus $\eta_{n, i} \sim \mathbf{p}(\zeta)$ for all $i$, and $Q_{n} \sim \mathbf{q}(\zeta)$ independent of $\left\{\eta_{n, i}: i \geq 1, n \geq 1\right\}$. Let $\mathfrak{F}_{n}$ denote the $\sigma$-field generated by $\left\{\zeta_{0}, \ldots, \zeta_{n}\right\}$, and let $\mathfrak{F}_{\infty}$ denote the $\sigma$-field generated by $\left\{\zeta_{0}, \zeta_{1}, \ldots\right\}$, and consider $V_{n}:=\mathbf{E}\left[Z_{n} \mid \mathfrak{F}_{\infty}\right]$. It is easily seen using the branching property that

$$
V_{n}=\mathbf{E}\left[\eta_{n, i} \mid \mathfrak{F}_{\infty}\right] V_{n-1}+\mathbf{E}\left[Q_{n} \mid \mathfrak{F}_{\infty}\right] .
$$

Assuming that $\mathbf{E}\left[\log \mathbf{E}\left[\eta_{n, 1} \mid \mathfrak{F}_{n}\right]\right]<0$ and letting $n \rightarrow \infty$ in the above equation, one obtains the linear recursion

$$
V \stackrel{d}{=} A V+B,
$$

where $V:=\lim _{n \rightarrow \infty} V_{n}$. A multidimensional extension of this model was the focus of the well-known paper of Kesten [22].

The recursion (34) also appears in many other settings, including the ARCH(1) and $\operatorname{GARCH}(1,1)$ processes used for financial time series modeling ( $c f$. [15], [5]), or the perpetuity sequences used for modeling the future liabilities of a life insurance company. For details, see [8], Section 3.

It is worth noting that in all of the above examples, it can be shown that the conjugate sequence in Theorem 2.2 may be taken to be zero, and thus the constant $C$ is determined solely by a perpetuity sequence which is killed in the event that $\left\{V_{n}\right\}$ returns, in the $\xi$-shifted measure, to its regeneration set; $c f$. [11], Corollary 2.1.

We conclude this section by remarking that in several real applications in the stationary case, simulation methods are typically used to obtain the tail probabilities, and this can be computationally expensive. Thus, a precise description of the tails of $V$ facilitates inference concerning the extreme percentiles of the distribution of $V$. Such estimates are of considerable interest in risk management. Estimation of $\xi$ has received much attention in the literature in risk theory, and detailed information concerning the Edgeworth expansion is available (see for instance [6]). However, 
inference concerning $C$ and the pair $(C, \xi)$ are not addressed in the literature. In an ongoing work, we use the change of measure arguments developed in [11] to describe a method-of-moments approach to the joint estimation of $(C, \xi)$. It turns out that in finite samples, the correlation between estimates of $C$ and $\xi$ is negative, and a detailed mathematical description is currently being carried out in [10].

\section{Recursions driven by Markov-dependent sequences}

Characterizing the constant $C$ in the Markov case is much more challenging than the i.i.d. case. As explained in Section 1, the values of the constant $C$ depend on the entire driving sequence $\left\{\left(\log A_{n}, B_{n}, D_{n}\right): n=1,2, \ldots\right\}$ and their inherent dependence structure. In the i.i.d. case, the recursions simplify, but in the Markov case, an important asymmetry is introduced between the forward and backward sequences, which we explain below in Section 3.1.

In spite of this complication, it is possible to adopt some of the principles from the i.i.d. case by utilizing the the regeneration technique of Athreya-Ney-Nummelin ([4], [28]), which states that the process contains independent blocks of random length which are i.i.d. Using this observation, we may derive appropriate SFPEs for Markov recursions and apply the results of the previous section. This is possible since, somewhat unexpectedly, the $k$-step composition of a recursion driven by Letac's Model E retains the general form of Letac's Model E, but with a new driving sequence (which here will be indexed by the regeneration times of the Markov chain $\left\{X_{n}\right\}$ ).

While the form of this constant will necessarily be complicated, we note in Remark 1 below that this constant reduces to the same general form as in the i.i.d. case in some important examples.

\subsection{Forward and backward Markov sequences}

Consider the forward and backward sequences associated with the SFPE $F_{Y}(v)=$ $A \max \{v, D\}+B$, where $Y \equiv(\log A, B, D)$. By (10), the forward sequence is given by

$$
V_{n}=A_{n} \max \left\{V_{n-1}, D_{n}\right\}+B_{n}, \quad n=1,2, \ldots, \quad V_{0}=v,
$$

while by (11), the backward sequence is given by

$$
Z_{n}=A_{1} \max \left\{Z_{n-1}^{(1)}, D_{1}\right\}+B_{1}, \quad n=1,2, \ldots, \quad Z_{0}=z,
$$

where $Z_{n-1}^{(1)}$ is defined as $Z_{n-1}$, but with $\left\{Y_{1}, \ldots, Y_{n-1}\right\}$ replaced with $\left\{Y_{2}, \ldots, Y_{n}\right\}$, i.e., the driving sequence is shifted forward by one unit of time. In contrast to the previous sections, we now assume that this driving sequence is Markov-dependent, 
that is, $Y_{n}=g\left(X_{n}\right)$, where $\left\{X_{n}\right\}$ is a Markov chain taking values in a state space $(\mathbb{S}, \mathscr{S})$ and $g: \mathbb{S} \rightarrow \mathbb{R}^{3}$. We will assume throughout this discussion that $\left\{X_{n}\right\}$ is aperiodic, irreducible (with respect to its maximal irreducibility measure $\varphi$ ), and countably generated. Thus, we adopt the basic set-up described in [29] or [25].

Markov-dependent forward and backward sequences arise widely in applications. Natural examples in the forward case include the $\mathrm{GARCH}(1,1)$ or $\mathrm{ARCH}(1)$ processes or branching processes with Markov-dependent innovations. While such examples are easily motivated in the case of the forward sequence (35), the utility of the backward sequence (36) is less transparent but can be motivated by a couple of elementary examples.

To this end, we return to the ruin problem with investments described in Example 2.2. In that example, we observed that the probability of ruin is $\mathbf{P}\{\mathscr{L}>u\}$, where $\mathscr{L} \equiv \sup _{n \in \mathbb{Z}_{+}} \mathscr{L}_{n}$ and $\mathscr{L}_{n}$ denotes the discounted losses of the company which accumulate by time $n$. Now by iterating the sequence $\left\{\mathscr{L}_{n}\right\}$, we obtain after an elementary argument that

$$
\mathscr{L}_{n}=A \max \left\{\mathscr{L}_{n-1}^{(1)}, 0\right\}+A L
$$

where $\mathscr{L}_{n-1}^{(1)}$ denotes that the driving sequence has been shifted forward by one unit in time; $c f$. the discussion following (33). Now if we set the initial state $z=0$ and $(B, D)=(A L, 0)$, then this last equation assumes the same form as (36), and our objective is to determine the maximum of the backward sequence $\left\{\mathscr{L}_{n}\right\}$.

A second example is the classical ruin problem mentioned in Example 2.1. In that example, the corresponding backward process is the multiplicative random walk $\mathscr{W}_{n}:=A_{1} \cdots A_{n}$ (where $A_{i}=\exp X_{i}$ is defined as in Example 2.1), and ruin can be shown to occur when $\tilde{\mathscr{L}}>e^{u}$, where

$$
\tilde{\mathscr{L}}:=\sup _{n \in \mathbb{Z}_{+}} \tilde{\mathscr{L}}_{n} \quad \text { and } \quad \tilde{\mathscr{L}}_{n}:=\max \left\{\mathscr{W}_{1}, \ldots, \mathscr{W}_{n}\right\}
$$

By repeating the above argument, we obtain that

$$
\tilde{\mathscr{L}}_{n}=A \max \left\{\tilde{\mathscr{L}}_{n-1}^{(1)}, 1\right\},
$$

which has the same form as (36) after setting $z=1$ and $(B, D)=(0,1)$.

In the above examples we see, rather generally, that forward sequences often arise in problems involving the steady-state limit of a given recursion, while backward sequences typically arise in problems involving maxima. Heuristically, these can be viewed as dual problems in the sense of Siegmund [31] or Asmussen and Sigman [3].

To analyze the processes (35) and (36), we again utilize the regeneration technique of Athreya-Ney-Nummelin, applied to the Markov chain $\left\{X_{n}\right\}$ (rather than to $\left\{V_{n}\right\}$ ), to derive an SFPE having the same form as (12); thus, in particular, Theorem 2 can be applied to describe the stationary limiting behavior, also in the Markovmodulated case. 
Let $P$ denote the transition kernel of $\left\{X_{n}\right\}$, and introduce the minorization condition

$$
h(x) \eta(E) \leq P(x, E), \quad x \in \mathbb{S}, E \in \mathscr{S},
$$

where $h(x)=\delta_{0} \mathbf{1}_{\mathscr{C}_{0}}(x)$ for some nontrivial set $\mathscr{C}_{0}$ and positive constant $\delta_{0}$, where $\eta$ is a probability measure on $(\mathbb{S}, \mathscr{S})$.

The regeneration lemma ([4], [28]) yields the existence of a sequence of stopping times $K_{0}, K_{1}, \ldots$ such that

(i) $\kappa_{i}:=K_{i}-K_{i-1}$ is an i.i.d. sequence, $i \geq 1$;

(ii) $\left\{\left(X_{K_{i-1}}, Y_{K_{i-1}}\right), \ldots,\left(X_{K_{i}-1}, Y_{K_{i}-1}\right)\right\}$ form independent blocks, $i \geq 1$.

(iii) $X_{K_{i}} \sim \eta$, independent of the past.

A standard calculation shows that both the recursions (35) and (36) have a nice compositional property, namely, if we calculate the $k$-step evolution of the process, then it can be viewed as a recursion involving the function $F_{Y}(v)=A \max \{v, D\}+B$, but with $Y_{n} \equiv\left(\log A_{n}, B_{n}, D_{n}\right)$ replaced with a new driving sequence. Specifically, after a tedius computation, we obtain that the $k$-step evolution satisfies

$$
V_{k}=\max \left\{\hat{\mathscr{A}} V_{0}, \hat{\mathscr{D}}\right\}+\hat{\mathscr{B}}, \quad k \in \mathbb{Z}_{+},
$$

where

$$
\begin{aligned}
\hat{\mathscr{A}} & :=A_{1} \cdots A_{k}, \\
\hat{\mathscr{B}} & :=\sum_{i=1}^{k} B_{i}\left(A_{i+1} \cdots A_{k}\right), \\
\hat{\mathscr{D}} & :=\bigvee_{j=1}^{k}\left[D_{j}\left(A_{j} \cdots A_{k}\right)-\sum_{i=1}^{j-1} B_{i}\left(A_{i+1} \cdots A_{k}\right)\right]
\end{aligned}
$$

(where $A_{1} \cdots A_{j-1}=1$ when $j=1$ ). Next observe that (37) has the same general form as (35), but with $(A, B, A D)$ replaced with $(\hat{\mathscr{A}}, \hat{\mathscr{B}}, \hat{\mathscr{D}})$. A similar expression is also obtained for the backward recursion.

This compositional property now carries over to the stopping times $K_{i}-1$. Thus, in the case of the forward recursion, we obtain for $\mathscr{V}_{i}:=V_{K_{i}-1}$ that

$$
\mathscr{V}_{i}=\max \left\{\mathscr{A}_{i} \mathscr{Y}_{i-1}, \mathscr{D}_{i}\right\}+\mathscr{B}_{i}, \quad i=1,2, \ldots
$$

In this recursion, the driving sequence $\mathscr{Y}_{i}:=\left(\log \mathscr{A}_{i}, \mathscr{B}_{i}, \mathscr{D}_{i}\right)$ is defined as $\hat{\mathscr{Y}}:=$ $(\log \hat{\mathscr{A}}, \hat{\mathscr{B}}, \hat{\mathscr{D}})$ in $(37)$, except that the deterministic interval $[1, k]$ in (37) must be replaced with the random interval $\left[K_{i-1}, K_{i}-1\right]$ in (38). (When $i=0, K_{i-1} \equiv 1$ in these definitions.) Thus, 


$$
\begin{aligned}
& \mathscr{A}_{i}:=A_{K_{i-1}} \cdots A_{K_{i}-1}, \\
& \mathscr{B}_{i}:=\sum_{j=K_{i-1}}^{K_{i}-1} B_{j}\left(A_{j+1} \cdots A_{K_{i}-1}\right), \\
& \mathscr{D}_{i}:=\bigvee_{j=K_{i-1}}^{K_{i}-1}\left[D_{j}\left(A_{j} \cdots A_{K_{i}-1}\right)-\sum_{k=K_{i-1}}^{j-1} B_{k}\left(A_{k+1} \cdots A_{K_{i}-1}\right)\right] .
\end{aligned}
$$

From (38), we obtain that $\mathscr{V}:=\lim _{i \rightarrow \infty} \mathscr{V} / i$ satisfies the SFPE

$$
\mathscr{V} \stackrel{d}{=} \max \{\mathscr{A} \mathscr{V}, \mathscr{D}\}+\mathscr{B}
$$

where $(\mathscr{A}, \mathscr{B}, \mathscr{D}) \stackrel{d}{=}\left(\mathscr{A}_{i}, \mathscr{B}_{i}, \mathscr{D}_{i}\right)$, and, as we will observe more formally below, $\left\{\mathscr{V}_{i}\right\}$ has the same steady-state limit as the original process $\left\{V_{n}\right\}$ and, thus, this steadystate limit is characterized as the solution to (40).

In the case of the backward sequence, the regeneration technique works similarly. It is just a matter of writing down the iterates, but now backward in time, to obtain for $\mathscr{Z}_{i}:=\max \left\{Z_{n}: 0 \leq n \leq K_{i}-1\right\}$ that

$$
\mathscr{Z}_{i}=\max \left\{\mathscr{A}_{1} \mathscr{Z}_{i-1}^{(1)}, \mathscr{D}_{1}\right\}+\mathscr{B}_{1}, \quad i=1,2, \ldots,
$$

where, following our usual convention, $\mathscr{Z}_{i-1}^{(1)}$ has the same distribution as $\mathscr{Z}_{i-1}$ but with the relevant driving sequence shifted forward by one unit in time, and for each positive integer $i$,

$$
\begin{aligned}
\mathscr{A}_{i} & :=A_{K_{i-1}} \cdots A_{K_{i}-1}, \\
\mathscr{B}_{i} & :=\sum_{j=K_{i-1}}^{K_{i}-1}\left(A_{K_{i-1}} \cdots A_{j-1}\right) B_{j}, \\
\mathscr{D}_{i} & :=\bigvee_{j=K_{i-1}}^{K_{i}-1}\left[\left(A_{K_{i-1}} \cdots A_{j}\right) D_{j}-\sum_{k=j+1}^{K_{i}-1}\left(A_{K_{i-1}} \cdots A_{k-1}\right) B_{k}\right] .
\end{aligned}
$$

(Once again, when $i=0, K_{i-1} \equiv 1$ in these definitions.)

Setting $\mathscr{Z}=\sup _{i} \mathscr{Z}_{i}$ in (41), we obtain the SFPE

$$
\mathscr{Z} \stackrel{d}{=} \max \{\mathscr{A} \mathscr{Z}, \mathscr{D}\}+\mathscr{B},
$$

where $(\mathscr{A}, \mathscr{B}, \mathscr{D}) \stackrel{d}{=}\left(\mathscr{A}_{i}, \mathscr{B}_{i}, \mathscr{D}_{i}\right)$.

It is important to observe that we obtain different distributions for $(\mathscr{B}, \mathscr{D})$ in the forward and backward cases, even though we have started with the same recursion, $F_{Y}(v)=A \max \{v, D\}+B$, to generate the sequences (35) and (36). Thus, there is an asymmetry between the forward and backward sequences, although the multiplicative term $\mathscr{A}$-which determines the polynomial rate of decay-remains the 
same. (We note that this feature appears even in the polynomial models with i.i.d. recursions, as described in [11], Example 3.5.)

We may now apply Theorems 2.2 and 2.3 directly to the SFPEs (38) and (43), but before doing so we will need to verify that the required moment conditions are satisfied. Since our random variables are formed over regeneration cycles, this issue is somewhat subtle and we address it in the next section.

\subsection{Characterizing moments over regeneration cycles}

\subsubsection{Moment properties of $\mathscr{A}$}

Let $g_{A}\left(X_{n}\right)=\log A_{n}$, and for each $\alpha \in \mathbb{R}$ define

$$
\hat{P}_{\alpha}(x, E):=\int_{E} e^{\alpha g_{A}(x)} P(x, d y) \quad \text { and } \quad \hat{P}_{\alpha}^{k}=\hat{P}_{\alpha} \circ \hat{P}_{\alpha}^{k-1}, \quad k>1 .
$$

Let $(\lambda(\alpha))^{-1}$ denote the convergence parameter of the kernel $\hat{P}_{\alpha}$ (for the definition, see [29], p. 27), and let $\Lambda(\alpha)=\log \lambda(\alpha)$. Set $S_{n}=\sum_{i=1}^{n} \log A_{i}$, and define

$$
\Gamma(\alpha)=\limsup _{n \rightarrow \infty} \frac{1}{n} \log \mathbf{E}\left[e^{\alpha S_{n}}\right], \quad \alpha \in \mathbb{R} .
$$

Roughly, the convergence parameter measures the growth rate of $\hat{P}_{\alpha}^{k}(x, E)$ as $k \rightarrow$ $\infty$, where $E$ is a "small set" satisfying $\left(\mathscr{M}_{0}\right)$, while the "Gärtner-Ellis" limit $\Gamma(\alpha)$ measures the growth rate of this quantity when $E=\mathbb{S}$. It is well known that $\Lambda(\alpha) \leq$ $\Gamma(\alpha)$ for all $\alpha$ ([27], [8]). Now assume that

$$
\Gamma(\xi)=0, \text { for some } \xi \in(0, \infty) \cap \operatorname{dom} \Lambda^{\prime} .
$$

Then it follows after a short argument that $\Lambda(\xi)=0$; see [8], p. 1426. Then by [27], we have under appropriate conditions that $1=\mathbf{E}\left[\left(A_{K_{i-1}} \cdots A_{K_{i}-1}\right)^{\xi}\right]:=\mathbf{E}[\mathscr{A} \xi]$. (See, in particular, Lemma 4.1 of [27] and its proof.) Thus, the solution $\xi$ to (44) yields the polynomial decay rate in Theorem 2.2, provided that appropriate moment conditions are satisfied.

Consequently, we obtain an explicit characterization of the decay constant in the Markov case, which is now the solution to the equation $\Lambda(\xi)=0$, but where the cumulant generating function is replaced with the function $\Lambda$ now derived from the convergence parameter or, alternatively, with the Gärtner-Ellis limit (as would be expected from the large deviation theory for Markov chains). 


\subsubsection{Moment properties of $(\mathscr{B}, \mathscr{D})$ : preliminary considerations}

For notational convenience, assume that regeneration occurs at time zero, and let $\kappa$ denote the subsequent regeneration time. Then, using the above expressions for $(\mathscr{B}, \mathscr{D})$ we see that in the forward case, the required moment conditions $\left(H_{2}\right)$ will be satisfied provided that $\mathbf{E}\left[\mathscr{B}_{f}^{\xi}\right]<\infty$, where

$$
\mathscr{B}_{f}:=\sum_{i=0}^{\kappa-1} \tilde{B}_{i}\left(A_{i+1} \cdots A_{\kappa-1}\right), \quad \tilde{B}_{i}:=\left|B_{i}\right|+\left|A_{i} D_{i}\right|
$$

and in this expression, we take $\left(A_{0}, B_{0}, D_{0}\right)$ to have the distribution of this triplet upon regeneration. Similarly, in the backward case, it is sufficient to verify that $\mathbf{E}\left[\mathscr{B}_{b}^{\xi}\right]<\infty$, where

$$
\mathscr{B}_{b}:=\sum_{i=0}^{\kappa-1}\left(A_{0} \cdots A_{i-1}\right) \tilde{B}_{i}, \quad \tilde{B}_{i}=\left|B_{i}\right|+\left|A_{i} D_{i}\right|
$$

These last two equations are manifestations of nearly the same mathematical quantity, as can be seen by constructing the time-reversed Markov process (whose existence is assured by [26]). Thus we extend $\left\{V_{n}: n \in \mathbb{N}\right\}$ to a doubly-infinite sequence $\left\{V_{n}: n \in \mathbb{Z}\right\}$, where these two sequences are identical for $n \in \mathbb{N}$. Then, by comparing (45) to this same quantity but over its prior regeneration cycle-that is, a cycle commencing at time $\tilde{\kappa}<0$ and terminating at time 0 -we obtain that

$$
\mathscr{B}_{f} \stackrel{d}{=} \sum_{i=1}^{-\tilde{\kappa}} \tilde{B}_{i}\left(A_{i+1} \cdots A_{1}\right)
$$

As with $\mathscr{B}_{b}$, this quantity may be viewed as a perpetuity sequence, but now computed backward in time (and shifted by one time unit compared with (46)). Consequently, the mathematical analysis is similar for the forward and backward sequences and, to avoid repetition, we will focus on verifying moment conditions for backward sequences in the sequel.

\subsubsection{The moments of $\mathscr{B}_{b}$ under some simplifying assumptions}

Our next objective is to relate the moments of $\mathscr{B}_{b}$ to the moments of the regeneration times of the $\xi$-shifted Markov chain, whose finiteness would then be assured if the Markov chain were $(\lambda(\alpha))^{-1}$-geometrically recurrent with $\alpha=\xi$. In the interest of simplicity, we will first develop this correspondence under a number of simplifying assumptions and later indicate how these assumptions can be removed.

Assume, for the moment, that $D_{i} \equiv 0$ for all $i$ and that $\left\{B_{i}\right\}$ is an i.i.d. sequence which is independent of the Markov-dependent sequence $\left\{A_{i}\right\}$. Next, introduce the 
strong minorization condition

$$
a \eta(E) \leq P(x, E), \quad x \in \mathbb{S}, E \in \mathscr{S}
$$

where $a>0$ and $\eta$ is a probability measure.

If the kernel $\hat{P}_{\alpha}$ is $(\lambda(\alpha))^{-1}$-recurrent, then there exists a right invariant function $r_{\alpha}$ satisfying the equation $\hat{P}_{\alpha} r_{\alpha}=\lambda(\alpha) r_{\alpha}$. Otherwise, $\hat{P}_{\alpha}$ is $(\lambda(\alpha))^{-1}$-transient and there exists a right subinvariant function $r_{\alpha}$ ([29], Section 5.1). For any $\alpha \in \operatorname{dom}(\lambda)$, introduce the $\alpha$-shifted transition kernel

$$
Q_{\alpha}(x, E)=\int_{E} \frac{e^{\alpha g_{A}(x)} r_{\alpha}(y)}{\lambda(\alpha) r_{\alpha}(x)} P(x, d y), \quad x \in \mathbb{S}, E \in \mathscr{S} .
$$

Then $Q_{\alpha}$ is a probability kernel when $\hat{P}_{\alpha}$ is $(\lambda(\alpha))^{-1}$-recurrent (and a subprobability kernel in the transient case). Let $\mathbf{E}_{\alpha}[\cdot]$ denote expectation with respect to this shifted measure.

Observe that the minorization $\left(\mathscr{M}_{0}\right)$ (or the stronger condition $\left(\mathscr{M}_{1}\right)$ ) induces a minorization for $Q_{\alpha}$; in particular, using the definition of $Q_{\alpha}$ together with $\left(\mathscr{M}_{0}\right)$, we obtain

$$
h_{\alpha}(x) \eta_{\alpha}(E) \leq Q_{\alpha}(x, E), \quad x \in C, E \in \mathscr{S},
$$

where, for some normalizing constant $L$,

$$
h_{\alpha}(x)=\frac{L h(x)}{\lambda(\alpha) r_{\alpha}(x)} e^{\alpha g_{A}(x)} \wedge 1 \quad \text { and } \quad \eta_{\alpha}(d y)=\frac{1}{L} r_{\alpha}(y) \eta(d y) .
$$

Here $L$ is a normalizing constant, chosen such that $\eta_{\alpha}$ is a probability measure. (We may assume that $\eta$ has been selected in a suitable way so that $L<\infty$.) Thus, a minorization exists, and hence a regeneration structure for the $\xi$-shifted chain. Also set $\hat{h}_{\alpha}(x)=h(x) e^{\alpha g_{A}(x)} / \lambda(\alpha)$.

Lemma 1 Assume $\left\{B_{i}\right\}$ is i.i.d. and independent of $\left\{A_{i}\right\}$ with $\mathbf{E}\left[B_{i}^{\xi}\right]<\infty$, and assume that $D_{i} \equiv 0$ for all $i$ and $\left(\mathscr{M}_{1}\right)$ is satisfied. Then

$$
\mathbf{E}_{\xi}[\kappa]<\infty \Longrightarrow \mathbf{E}\left[\mathscr{B}_{b}^{\xi}\right]<\infty .
$$

Proof. For the proof, introduce the notation $h \otimes \eta(x, d y):=h(x) \eta(d y)$.

Using the series representation for a regeneration cycle (as in [28], p. 313 or Lemma 4.1 of [27]), we obtain 


$$
\begin{aligned}
\mathbf{E}_{\xi}\left[\kappa-1 \mid X_{0}=x\right] & =\mathbf{E}_{\xi}\left[\sum_{n=1}^{\infty} \mathbf{1}_{\{\kappa>n\}} \mid X_{0}=x\right] \\
& =\sum_{n=1}^{\infty} \int_{\mathbb{S}}\left(Q_{\xi}-h_{\xi} \otimes \eta_{\xi}\right)^{n}(x, d y) \\
& \geq \frac{1}{r_{\xi}(x)} \sum_{n=1}^{\infty}\left(\hat{P}_{\xi}-\hat{h}_{\xi} \otimes \eta\right)^{n} r_{\xi}(x) \\
& =\frac{1}{r_{\xi}(x)} \sum_{n=1}^{\infty} \mathbf{E}\left[e^{\left.\xi S_{n-1}^{\prime} \mathbf{1}_{\{\kappa>n\}} r_{\xi}\left(X_{n}\right) \mid X_{0}=x\right]}\right.
\end{aligned}
$$

where $S_{n}^{\prime}:=\sum_{i=0}^{n} \log A_{i}$. In the previous display, the inequality follows directly from the definitions of $h_{\xi}, \hat{h}_{\xi}, \eta_{\xi}, \hat{P}_{\xi}$, and $Q_{\xi}$ (and we obtain an inequality here due to the additional term " $\wedge 1$ " appearing in the definition of $h_{\xi}$ ).

Next observe that under the strong minorization $\left(\mathscr{M}_{1}\right)$, the function $r_{\xi}$ is bounded below by a constant ([7], Remark 2.3). It follows that

$$
\mathbf{E}\left[\sum_{n=1}^{\infty}\left(A_{0} \cdots A_{n-1}\right)^{\xi} \mathbf{1}_{\{\kappa>n\}} \mid X_{0}=x\right] \leq M r \xi(x), \quad \text { for some } M<\infty .
$$

Using independence and the moment assumption on $\left\{B_{i}\right\}$, we conclude that this expression also holds with $\left(A_{0} \cdots A_{n-1} B_{n}\right)$ in place of $\left(A_{0} \cdots A_{n-1}\right)$ and $M$ replaced with some finite constant $M^{\prime}$. (Since $\left\{B_{i}\right\}$ is i.i.d. and independent of $\left\{A_{i}\right\}$, the $B$ sequence is independent of the regeneration times.) Consequently,

$$
\mathbf{E}\left[\mathscr{B}_{b}^{\xi}\right] \leq M^{\prime} \int r_{\xi}(x) \eta(d x)
$$

In the minorization $\left(\mathscr{M}_{1}\right)$, we may assume that the measure $\eta$ has been chosen such that the integral on the right-hand side of the last expression is finite. Thus we obtain $\mathbf{E}\left[\mathscr{B}_{b}^{\xi}\right]<\infty$, as required.

\subsubsection{The moments of $\mathscr{B}_{b}$ in the general case}

The previous argument can be modified to incorporate a nontrivial sequence $\left\{D_{i}\right\}$ and Markov dependence in the entire driving sequence $\left\{\left(\log A_{i}, B_{i}, D_{i}\right)\right\}$. Following Collamore ([8], Section 6.1), one approach is to replace the kernel $\hat{P}_{\xi}$ in the above argument with $\hat{R}_{\xi}$, where for any $\alpha$,

$$
\hat{R}_{\alpha}(x, E):=\int_{E} e^{\alpha F(x, y)} P(x, d y), \quad x \in \mathbb{S}, E \in \mathscr{S},
$$

for $\left(f_{A}\left(X_{n}\right), f_{B}\left(X_{n}\right)\right)=\left(\log A_{n}, \log \left(\tilde{B}_{n}+1\right)\right)$ and 


$$
F(x, y)=f_{A}(x)+\left(f_{B}(y)-f_{B}(x)\right) .
$$

If the minorization has been chosen so that $\tilde{B}_{0}$ is deterministically bounded from above by a constant, then the previous lemma can be repeated to obtain the same result as before, although the $Q$-shifted measure is formed with respect to the kernel $\hat{R}_{\xi}$ rather than $\hat{P}_{\xi}$. That is to say, we now define

$$
\tilde{Q}_{\alpha}(x, E)=\int_{E} \frac{e^{\alpha F(x, y)} \tilde{r}_{\alpha}(y)}{\tilde{\lambda}(\alpha) \tilde{r}_{\alpha}(x)} P(x, d y), \quad x \in \mathbb{S}, E \in \mathscr{S},
$$

where $\tilde{r}_{\alpha}$ and $\tilde{\lambda}(\alpha)$ are the eigenvectors and eigenvalues corresponding to the kernel $\hat{R}_{\alpha}$. Let $\tilde{\mathbf{E}}_{\alpha}[\cdot]$ denote expectation with respect to this measure. In addition, assume that $\Gamma(\xi)=\tilde{\Gamma}(\xi)=0$, where

$$
\tilde{\Gamma}(\alpha):=\limsup _{n \rightarrow \infty} \frac{1}{n} \log \mathbf{E}\left[e^{\alpha S_{n}}\left(\tilde{B}_{n}+1\right)^{\alpha}\right] .
$$

Then we obtain:

Lemma 2 Assume $\left(\mathscr{M}_{1}\right)$. Then

$$
\tilde{\mathbf{E}}_{\xi}[\kappa]<\infty \Longrightarrow \mathbf{E}\left[\mathscr{B}_{b}^{\xi}\right]<\infty .
$$

Finally, we observe that $\left(\mathscr{M}_{1}\right)$ may be weakened to $\left(\mathscr{M}_{0}\right)$ by first introducing the "augmented kernel"

$$
P_{a}(x, E):=P(x, E)+a \eta(E),
$$

and then computing the $\xi$-shifted measure using this kernel in place of $P$; cf. [7], [8]. Under this construction, the right invariant function $r_{\xi, a}$ is uniformly positive, as required in the proofs of Lemmas 3.1 and 3.2, and the eigenvalue $\lambda_{a}(\xi) \downarrow 1$ as $a \downarrow 0$.

\subsubsection{Toward a complete result in the Markov case}

The moment assumptions in Lemmas 3.1 and 3.2, expressed in terms of the $\xi$ shifted measures, are not particularly natural to verify in practice, where it would be preferable to express these conditions in terms of the transition kernel of the original process. Moreover, there is also a need to verify the further moment assumption on $\mathscr{A}$, equivalent to the assumption that $\mathbf{E}\left[\mathscr{A}^{\xi}(\log \mathscr{A})\right]<\infty$. Now under appropriate conditions, it is known ([27], pp. 581-2) that

$$
\mathbf{E}\left[\mathscr{A}^{\xi}(\log \mathscr{A})\right]=\mathbf{E}_{\xi}[\log \mathscr{A}]=\mathbf{E}_{\xi}[\kappa] \mathbf{E}_{\xi}[\log A \mid X \sim \pi],
$$

where $\pi$ denotes the stationary measure of $\left\{X_{n}\right\}$. 
Roughly speaking, a sufficient condition for the above results to hold is the geometric $\xi$-recurrence of the kernels $\hat{P}_{\xi}$ and $\hat{R}_{\xi}$ (cf. [29], Proposition 5.25). Thus, it is of some theoretical and applied interest to understand how geometric $\xi$-recurrence relates to the underlying properties of the given Markov chain.

An effort to draw this connection has been given in [8]. Let $\mathfrak{h}: \mathbb{S} \rightarrow[0, \infty)$, and define:

$$
\begin{aligned}
\mathscr{L}_{a} \mathfrak{h} & =\{x \in \mathbb{S}: \mathfrak{h}(x) \leq a\}, \quad a \geq 0 ; \\
\tilde{S}_{n} & =\left\{\log A_{1}+\cdots+\log A_{n-1}\right\}+\log \left(\tilde{B}_{n}+1\right), \quad n=1,2, \ldots ; \\
S_{n}^{\mathfrak{h}} & =\mathfrak{h}\left(X_{1}\right)+\cdots+\mathfrak{h}\left(X_{n}\right), \quad n=1,2, \ldots ; \\
\Gamma_{\mathfrak{h}}(\alpha, \beta) & =\limsup _{n \rightarrow \infty} \frac{1}{n} \log \mathbf{E}\left[e^{\alpha \tilde{S}_{n}+\beta S_{n}^{\mathfrak{h}}}\right], \quad(\alpha, \beta) \in \mathbb{R}^{2} .
\end{aligned}
$$

Assume the existence of a nonnegative $\mathfrak{h}$-function such that the following condition holds.

\section{Minorization:}

$(\mathfrak{M})$ For any $a>0$ sufficiently large, there exist a constant $\delta_{a}>0$ and a probability measure $\eta_{a}$ on $(\mathbb{S}, \mathscr{S})$ with $\eta_{a}\left(\mathscr{L}_{a} \mathfrak{h}\right)>0$ such that

$$
\delta_{a} \mathbf{1}_{\mathscr{L}_{a} \mathfrak{h}}(x) \eta_{a}(E) \leq P(x, E), \quad x \in \mathbb{S}, E \in \mathscr{S} .
$$

Also impose the following additional assumptions on the process.

Hypotheses:

$\left(\mathscr{H}_{1}\right)$ For the function $\mathfrak{h}$ given in $(\mathfrak{M})$, there exist points $\alpha>r$ and $\beta>0$ such that $\Gamma_{\mathfrak{h}}(\alpha, \beta)<\infty$.

$\left(\mathscr{H}_{2}\right)$ For any $a>0$, there exist nontrivial sets $E_{1}, \ldots, E_{l} \subset \mathbb{S}$, possibly dependent on $a$, and a finite constant $J_{a}$ such that

$$
P(x, E) \leq J_{a} \inf \left\{\sum_{i=1}^{l} P\left(x_{i}, E\right): x_{i} \in E_{i}, 1 \leq i \leq l\right\}, \quad x \in \mathscr{L}_{a} \mathfrak{h}, E \in \mathscr{S} .
$$

We also need to assume the usual regularity assumptions, now with respect the the random variables formed over a regeneration cycle. We collect these assumptions as an additional hypothesis:

$\left(\mathscr{H}_{3}\right)$ Hypotheses $\left(H_{0}\right)$ and $\left(H_{3}\right)$ hold with respect to $(\mathscr{A}, \mathscr{B}, \mathscr{D})$.

In Collamore [8], it is shown that if $(\mathfrak{M}),\left(\mathscr{H}_{1}\right)$, and $\left(\mathscr{H}_{2}\right)$ hold, then

$$
\mathbf{E}\left[\mathscr{A}^{\alpha}\right]<\infty \quad \text { and } \quad \mathbf{E}\left[\mathscr{B}_{b}^{\alpha}\right]<\infty, \quad \text { for some } \alpha>\xi .
$$

Moreover, $\mathbf{E}\left[\mathscr{B}_{f}^{\alpha}\right]<\infty$, provided that these conditions hold with respect to the timereversed Markov chain (which we implicitly assume in the following development when the forward recursion is considered). 
Note that the property described in $(\mathfrak{M})$ always holds for Harris chains when $P$ is replaced by $P^{k_{a}}$; see [25]. Also, we note that a regeneration structure we need would still exist if $\left(\mathscr{M}_{0}\right)$ were weakened to a condition on $P^{k}$ rather than $P$, that is, to Harris recurrence; see [29], p. 134. Finally, it seems plausible that condition $\left(\mathscr{H}_{2}\right)$ could possibly be removed, since this is mainly used in [8] to assure that the relevant eigenvectors are bounded from above by a constant.

Combining the results of [11] and [8], we obtain an extension of Theorem 2.2. First let $\left\{\mathscr{Z}_{n}^{(p)}\right\}$ and $\left\{\mathscr{Z}_{n}^{(c)}\right\}$ denote the perpetuity and conjugate sequences, defined as in (17) and (18), but with $\left(A_{i}, B_{i}, D_{i}\right)$ replaced with $\left(\mathscr{A}_{i}, \mathscr{B}_{i}, \mathscr{D}_{i}\right)$. Then set $\mathscr{Z}^{(p)}=\lim _{n \rightarrow \infty} \mathscr{Z}_{n}^{(p)}$ and $\mathscr{Z}^{(c)}=\lim _{n \rightarrow \infty} \mathscr{Z}_{n}^{(c)}$. Also, let $\left\{\tilde{\mathscr{V}}_{n}\right\}$ denote the forward process generated by the sequence $\left\{\mathscr{A}_{i}, \mathscr{B}_{i}, \mathscr{D}_{i}\right\}$ according to Letac's Model E, and set $\tau=\inf \left\{i: \tilde{\mathscr{V}}_{i} \in \mathscr{C}\right\}$, that is, the first passage time of this Markov chain into its $\mathscr{C}$-set. $\left(\tilde{\mathscr{V}}_{i} \equiv \mathscr{V}_{i}\right.$ if it is a forward process we study, but not if it is a backward process.) Finally, recall that $\lambda(\alpha)$ denotes the convergence parameter associated with the kernel $\hat{P}(\alpha)$, as defined in Section 3.2.1.

Theorem 5 Assume that (44) holds and that $(\mathfrak{M}),\left(\mathscr{H}_{1}\right),\left(\mathscr{H}_{2}\right)$, and $\left(\mathscr{H}_{3}\right)$ are satisfied. Then

$$
\lim _{u \rightarrow \infty} u^{\xi} \mathbf{P}\{W>u\}=C
$$

for a finite positive constant $C$, where $W:=\lim _{n \rightarrow \infty} V_{n}$ in the forward case and $W:=\sup _{n} Z_{n}$ in the backward case. Moreover, the constant $C$ may be identified as

$$
C=\frac{1}{\xi \lambda^{\prime}(\xi) \mathbf{E}[\tau]} \mathbf{E}_{\xi}\left[\left(\mathscr{Z}^{(p)}-\mathscr{Z}^{(c)}\right)^{\xi} \mathbf{1}_{\{\tau=\infty\}}\right] .
$$

Remark 1 In specific examples, the constant $C$ can be identified more explicitly and put into the same general form as in the i.i.d. case studied in Theorem 2.2. In particular, if $\left\{V_{n}\right\}$ is obtained from the forward recursion $V_{n}=A_{n} V_{n-1}+B_{n}$ and the sequence $\left\{B_{n}\right\}$ is supported on $[0, \infty)$, then the conjugate term in (50) is zero (since all nonzero terms in (18) would then be positive), and so it follows from (50) and (39) that

$$
\mathscr{Z}^{(p)}=V_{0}+\frac{B_{1}}{A_{1}}+\frac{B_{2}}{A_{1} A_{2}}+\cdots,
$$

where, as in Theorem 2.2, the initial distribution (corresponding to the distribution of the random variable $V_{0}$ ) is obtained from the regeneration measure of the Markov chain $\left\{V_{K_{i}}: i=0,1, \ldots\right\}$. We remind the reader that the sequence $\left\{K_{i}\right\}$ represents the regeneration times of the Markov chain $\left\{X_{n}\right\}$, and that $\left(A_{n}, B_{n}\right)$ is modulated by this chain $\left\{X_{n}\right\}$, that is, $\left(A_{n}, B_{n}\right)=g\left(X_{n}\right)$.

Moreover, the stopping time $\tau$ can also be viewed-as in Theorem 2.2-as a first passage time. Specifically, in Nummelin's split-chain construction ([28], [29]), regeneration of the Markov chain $\left\{X_{n}\right\}$ occurs when $Y_{n}:=\left(X_{n}, \gamma_{n}\right) \in \mathscr{C}_{0} \times\{1\}$, where $\left\{\gamma_{n}\right\}$ is an i.i.d. Bernoulli sequence with $\mathbf{P}\left\{\gamma_{n}=1\right\}=\delta_{0}$ and $\left(\mathscr{C}_{0}, \delta_{0}\right)$ appear in the minorization $\left(\mathscr{M}_{0}\right)$ of the Markov chain $\left\{X_{n}\right\}$. Now, $\{\tau=\infty\}$ corresponds to the event that $\left\{V_{K_{i}}\right\}$ never returns to its $\mathscr{C}$-set (namely, the interval $\mathscr{C}=[-M, M]$ ); that 
is to say, $\left\{V_{n}\right\}$ never returns to $\mathscr{C}$ at a regeneration time of $\left\{X_{n}\right\}$. But this is the same as the condition that $\left\{\left(V_{n}, Y_{n}\right): n=0,1, \ldots\right\}$ never returns to the set $\mathscr{C} \times \mathscr{C}_{0} \times\{1\}$.

Now if $\left\{V_{n}\right\}$ is a perpetuity sequence (thus obtained from backward recursion of the SFPE $f(v)=A v+B$ rather than forward recursion of this SFPE), then (42) must be employed in place of (39), which does not simplify in the same way as (51). However, in this case, it is plausible to employ [26] to obtain the time-reversed process of $\left\{\left(V_{n}, A_{n}, B_{n}\right): n=0,1, \ldots\right\}$, and to observe that this time-reversed process assumes the form of the forward sequence $V_{n}=\tilde{A}_{n} V_{n-1}+\tilde{B}_{n}$, where $\left(\tilde{A}_{n}, \tilde{B}_{n}\right)=\left(A_{-n}, B_{-n}\right)$ for the extended process $\left\{\left(A_{n}, B_{n}\right): n \in \mathbb{Z}\right\}$. Since the limiting distribution of this forward process agrees with the limiting distribution of the original perpetuity sequence, we expect an expression of the form (51), also for the case of perpetuities.

Remark 2 In [8], Section 3, the conditions of Theorem 3.1 are verified for a variety of problems which are of applied interest. One application considered in [8] is the ruin problem with investments (described above in Example 2.2), but where the investment returns are Markov-dependent, governed by any one of the following:

(i) A discrete-time Black-Scholes model under Markov regime switching, where the regime switching is determined by an underlying finite-state or uniformly recurrent Markov chain.

(ii) The logarithmic returns $\left\{-\log A_{i}\right\}$ are modeled as an $\operatorname{AR}(p)$ process or, with slight modifications of the assumptions, an $\operatorname{ARMA}(p, q)$ process.

(iii) The insurance company invests a fixed fraction of its surplus capital in a stock and a fixed fraction in a bank account, where the returns on the bank investment are at a deterministic rate $r>1$, while the returns on the stock investment follow the stochastic volatility model suggested in [13], [14]. Specifically, the investment returns are modeled as $R_{n}=\sigma_{n} \zeta_{n}$, where $\left\{\zeta_{n}\right\}$ is an i.i.d. Gaussian sequence and $\left\{\log \sigma_{n}\right\}$ is modeled, say, as an $\operatorname{ARMA}(p, q)$ process.

Another application considered in $[8]$ is a $\operatorname{GARCH}(1,1)$ process with regime switching, where the regime shifts are (as in (i)) modulated by an underlying finitestate Markov chain.

The proof of Theorem 3.1 is a direct consequence of Theorem 2.1 of [11] and Theorem 4.1 of [8]. In the forward case, it also needs to be observed that the limit over regeneration cycles agrees with the steady-state limit of the original sequence. But this equivalence is obtained along the lines of [8], p. 1428.

\section{References}

1. Alsmeyer, G., Iksanov, A., Rösler, U.: On distributional properties of perpetuities. J. Theor. Probability 22, 666-682 (2009)

2. Asmussen, S., Albrecher, H.: Ruin Probabilities, 2nd edn. World Scientific, River Edge, NJ (2010)

3. Asmussen, S., Sigman, K.: Monotone stochastic recursions and their duals. Probab. Th. Eng. Inf. Sc. 10, 1-20 (1996)

4. Athreya, K.B., Ney, P.: A new approach to the limit theory of recurrent Markov chains. Trans. Amer. Math. Soc. 245, 493-501 (1978) 
5. Bollerslev, T.: Generalized autoregressive conditional heteroskedasticity. J. Econometrics 31, 307-327 (1986)

6. Brito, M., Freitas, A.: Edgeworth expansion for an estimator of the adjustment coefficient. Insurance: Mathematics and Economics 43, 203-208 (2008)

7. Collamore, J.F.: Importance sampling techniques for the multidimensional ruin problem for general Markov additive sequences of random variables. Ann. Appl. Probab. 12, 382-421 (2002)

8. Collamore, J.F.: Random recurrence equations and ruin in a Markov-dependent stochastic economic environment. Ann. Appl. Probab. 19, 1404-1458 (2009)

9. Collamore, J.F., Diao, G., Vidyashankar, A.N.: Rare event simulation for processes generated via stochastic fixed point equations. Preprint (Math arXiv PR/1107.3284) (2011)

10. Collamore, J.F., Diao, G., Vidyashankar, A.N.: Joint inference for tail parameters of SFPE. In preparation (2013)

11. Collamore, J.F., Vidyashankar, A.N.: Tail estimates for stochastic fixed point equations via nonlinear renewal theory. Stoch. Process. Appl. 123, 3378-3429 (2013)

12. Cramér, H.: On the Mathematical Theory of Risk. In: Försäkringsaktiebolaget Skandia 18551930, Parts I and II, Stockholm 1930, 7-84 (1930)

13. Davis, R., Mikosch, T.: Extremes of stochastic volatility models. In: T.G. Andersen, R.A. Davis, J.P. Kreiss, T. Mikosch (eds.) Handbook of Financial Time Series. Springer Verlag, Berlin (2008)

14. Davis, R., Mikosch, T.: Probabilistic properties of stochastic volatility models. In: T.G. Andersen, R.A. Davis, J.P. Kreiss, T. Mikosch (eds.) Handbook of Financial Time Series. Springer Verlag, Berlin (2008)

15. Engle, R.F.: Autoregressive conditional heteroscedasticity with estimates of the variance of United Kingdom inflation. Econometrica 50, 987-1007 (1982)

16. Enriquez, N., Sabot, C., Zindy, O.: A probabilistic representation of constants in Kesten's renewal theorem. Probab. Theory Relat. Fields 144, 581-613 (2009)

17. Furstenberg, H.: Noncommuting random products. Trans. Amer. Math. Soc. 108, 377-428 (1963)

18. Goldie, C.M.: Implicit renewal theory and tails of solutions of random equations. Ann. Appl. Probab. 1, 126-166 (1991)

19. de Haan, L., Resnick, S., Rootzén, H., de Vries, C.G.: Extremal behaviour of solutions to a stochastic difference equation with applications to ARCH processes. Stoch. Process. Appl. 32, 213-224 (1989)

20. Hitczenko, P., Wesołowski, J.: Renorming divergent perpetuities. Bernoulli 17(3), 880-894 (2011)

21. Iglehart, D.L.: Extreme values in the GI/G/1 queue. Ann. Math. Statist. 43, 627-635 (1972)

22. Kesten, H.: Random difference equations and renewal theory for products of random matrices. Acta Math. 131, 207-248 (1973)

23. Letac, G.: A contraction principle for certain Markov chains and its applications. random matrices and their applications. Proceedings of AMS-IMS-SIAM Joint Summer Research Conference 1984. Contemporary Mathematics 50, 263-273 (1986)

24. Lundberg, F.: Approximerad Framställning av Sannolikhetsfunktionen. Återförsäkring av Kollektivrisker. Akad. Afhandling. Almqvist och Wiksell, Uppsala (1903)

25. Meyn, S., Tweedie, R.: Markov Chains and Stochastic Stability. Springer-Verlag, Berlin (1993)

26. Nelson, E.: The adjoint Markoff process. Duke Math. J. 25, 671-690 (1958)

27. Ney, P., Nummelin, E.: Markov additive processes I. Eigenvalue properties and limit theorems. Ann. Probab. 15, 561-592 (1987)

28. Nummelin, E.: A splitting technique for Harris recurrent Markov chains. Z. Wahrsch. Verw. Gebiete 43, 309-318 (1978)

29. Nummelin, E.: General Irreducible Markov Chains and Non-negative Operators. Cambridge University Press, Cambridge (1984)

30. Roitershtein, A.: One-dimensional linear recursions with Markov-dependent coefficients. Ann. Appl. Probab. 17, 572-608 (2007) 
31. Siegmund, D.: The equivalence of absorbing and reflecting barrier problems for stochastically monotone Markov processes. Ann. Probab. 4, 914-924 (1976)

32. Siegmund, D.: Note on a stochastic recursion. In: State of the art in probability and statistics (Leiden, 1999), IMS Lecture Notes Monogr. Ser., 36, pp. 547-554. Inst. Math. Statist., Beachwood, OH (2001)

33. Woodroofe, M.: Nonlinear Renewal Theory in Sequential Analysis. SIAM, Philadelphia (1982)

34. Yakin, B., Pollak, M.: A new representation for a renewal-theoretic constant appearing in asymptotic approximations of large deviations. Ann. Appl. Probab. 8, 749-774 (1998) 